The fabrication of nanoscale structures using the impact of multiply charged ions on solids is foreseen because although the ions are neutralized well above the surface most of their potential energy is still carried into the surface layers.

\title{
The Interaction of Highly Charged lons with Surfaces
}

A multiply charged ion approaching a solid surface can be regarded as a bomb containing a large amount of potential energy. This energy, comprising the sum of the atom's ionization potentials, is in principle available for inducing a variety of dynamic processes when the ion hits the surface (an $\mathrm{Ar}^{10+}$ ion, for example, contains roughly $1 \mathrm{keV}$ of potential energy). The main questions concern the mechanism by which this energy is dissipated and the effects of such a collision.

There has been a great deal of speculation about so-called Coulomb explosion processes which may be induced by the impact of a multiply charged ion, and the scenario envisaged is sketched in Fig. 1. When the projectile approaches the surface sufficiently slowly, electrons from the surface will be transferred to the ion leading to a depletion of electrons over a small area. If replacement of these electrons from the solid is not sufficiently fast, nuclei will be ejected from the surface due to their mutual Coulomb repulsion. Surface damage in a small but well-defined area can therefore result from the impact of slowly moving, highly charged ions. Since this damage will be very localized, the collision of multiply charged ions with surfaces can possibly be exploited to create nanostructures.

No experiment has so far yielded clear evidence for Coulomb explosion. This can be partly ascribed to the fact that metallic surfaces have mainly been used as targets for multiply charged ion bombardment. Since metals have very short electronic relaxation times, electrons are replaced from the bulk before Coulomb explosion can take place. However, a detailed understanding of the electronic processes that occur in the close vicinity of a surface has been obtained from various experiments made during the last few years using metallic surfaces. The information obtained by

Jayani Das has been with the research staff of the Max-Planck-Institut für Plasmaphysik, Garching bei München, since 1993. She studied at the Indian Institute of Technology, Kharagpur, and received her Ph.D. in nuclear physics from the State University of New York, Stony Brook, USA, in 1990 before working in atomic and surface physics for three years as a postdoc at the Kernfysisch Versneller Instituut, Rijksuniversiteit Groningen, NL-9747 AA Groningen.

Reinhard Morgenstern has been a full professor of atomic physics at Rijksuniversiteit Groningen since 1986. He studied at Freiburg University where he received his Ph.D. in 1971. After working as a postdoc at the Southern Research Institute, Menlo Park, USA, he joined the research staff of the University of Utrecht in 1979.

\section{R. Morgenstern, J. Das}

\author{
Kernfysisch Versneller Instituut, Groningen, The Netherlands
}

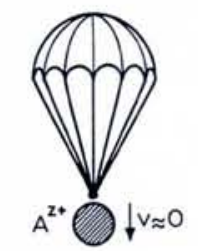

Fig. 1 - An illustration of Coulomb explosion arising during the interaction of a multiply charged ion with a surface. When an ion $A^{Z+}$ with a charge $Z$ slowly approaches the surface of a solid $B$, electrons are extracted from the surface and ions $\mathrm{B}^{+}$are subsequently ejected.

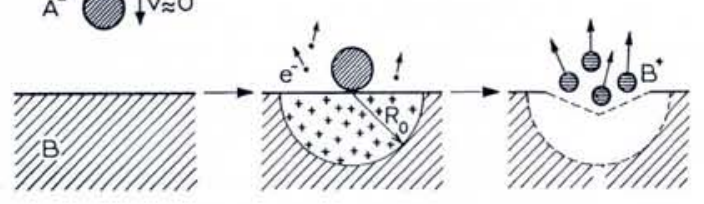

corresponding bending towards the surface does not occur, and the total scattering angle is therefore increased. Winter could therefore extract from measured total scattering angles the amount of

combining analyses of electrons and photons emitted during the collision with those of projectiles scattered at the surface allows us to reconstruct the history of the interaction. To a first order, one can distinguish three phases: a pre-collision phase, the touch-down, and a phase involving collisions inside the solid.

\section{The Pre-collision Phase}

It will be shown that many phenomena occur even before the ion actually hits the surface. In particular: an image charge is formed (at least in the case of metallic surfaces); electrons are transferred from the surface to the projectile giving rise to socalled neutral "hollow atoms"; and Auger processes within these hollow atoms lead to electron emission above the surface.

The image charge which forms initially involves rearrangement of the conduction electrons in the metal's surface such that a positive ion in front of the surface "sees" its own negative mirror image within the solid. This image charge causes an acceleration of the ions towards the surface. It also has a strong influence on the electrostatic potential in front of the surface, and hence on the motion of electrons in this region.

The acceleration induced by the image charge of the projectiles has been directly demonstrated by $\mathrm{H}$. Winter from Münster [1] who studied the scattering of highly charged ions from surfaces at grazing angles of incidence. The experiments exploited the fact that projectiles impinging at very small angles with respect to the surface are specularly reflected. For a neutral projectile, the total scattering angle is therefore twice the angle of incidence. However, in the case of a highly charged ion the effective angle of incidence is increased due to the action of the image charge, which bends the trajectory towards the surface on its incoming part. On the outgoing part of the trajectory - the projectile now being neutralized - a trajectory bending, and the corresponding energy gain of the projectiles, due to the action of the image charge.

The image charge disappears as soon as the projectile is neutralized and the acceleration therefore ceases at some point. The position at which this occurs can be determined by inspecting a diagram of the electrostatic potential seen by electrons in front of the surface [2]. An adiabatic approximation for this potential is justified since the velocity of ions with $\mathrm{keV}$ energies is small compared to the rate of rearrangement of electrons near a metallic surface. The potential contains contributions from the ion charge, the ion's image charge, and from the image charge of the electron itself. The calculated potential in front of the surface is shown in Fig. 2 for an ion with charge $q=7$.

There exists between the surface and the ion an electrostatic potential barrier which

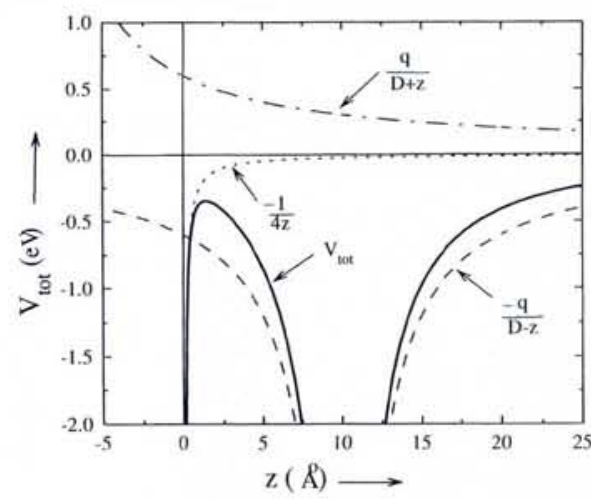

Fig. 2 - The electrostatic potential (solid line) "seen" by an electron in front of a metallic surface for an ion at a distance $D$ from the surface. The potential $V$ is plotted as a function of the distance above the surface and the contributions from the ion charge, the ion's image charge and the image charge of the electron are given by the dashed lines. 
decreases when the ion approaches the surface. At a critical distance given in atomic units by $D_{C}=(2 q+1 / 2)^{1 / 2} / W$ where $W$ represents the work function of the metal, the potential drops below the Fermi level of the metal. As soon as the condition is fulfilled electrons can move over from the metal to the projectile. This happens at a distance $D_{c}$ of about $10 \AA$ for a $\mathrm{N}^{7+}$ ion in front of a surface with a work function $W$ of $5 \mathrm{eV}$. Assuming instantaneous neutralization at $D_{c}$, the result is an increase in the kinetic energy gain of the projectile owing to image charge acceleration being proportional to $q^{2 / 3}$. It is remarkable that the energy gains determined experimentally by Winter are in nearly perfect agreement with theoretical estimates. This can be regarded as a direct validation of the classical model based on electron transfer and the nearly instantaneous neutralization at $D_{\mathrm{c}}$.

\section{Hollow atoms}

It is expected from the classical picture that electron transfer occurs quasi-resonantly into excited projectile levels (for $\mathrm{N}^{7+}$ ions, for example, initially into levels with principal quantum number $n=7$ ), eventually leading to a completely neutralized hollow atom with many electrons in excited levels and empty or sparsely populated inner levels. From the point of view of atomic physics, such multiply excited hollow atoms represent interesting objects whose properties are not yet well known. From the point of view of ion-surface interactions, the formation of hollow atoms implies that the ionic "bomb", although "neutralized", still contains most of its initial potential energy.

The formation of the hollow atom marks the start of a decay cascade proceeding via Auger or radiative processes which results in the emission of electrons and photons. The ion is neutralized again by capture of another electron from the surface every time an electron is emitted. However, only the short time interval between the capture of the first electron at $D_{c}$ and touch down on the surface is available for such a decay cascade. For instance, in the case of $\mathrm{N}^{7+}$ ions with a kinetic energy of $100 \mathrm{eV}$, the interval lasts only $27 \mathrm{fs}$. The decay cascade will therefore in general be incomplete at the time of touch down.

\section{Energy spectra}

Various experimental techniques can be used to gain insight into the processes

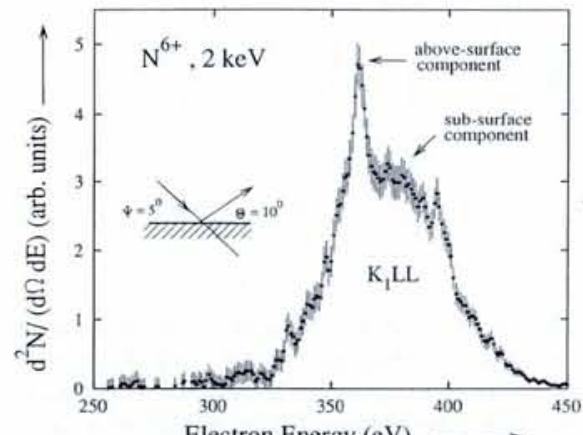

Fig. 3 - The energy spectrum of electrons emitted during collisions of $2 \mathrm{keV} \mathrm{N}^{6+}$ ions with a Ni(110) surface.
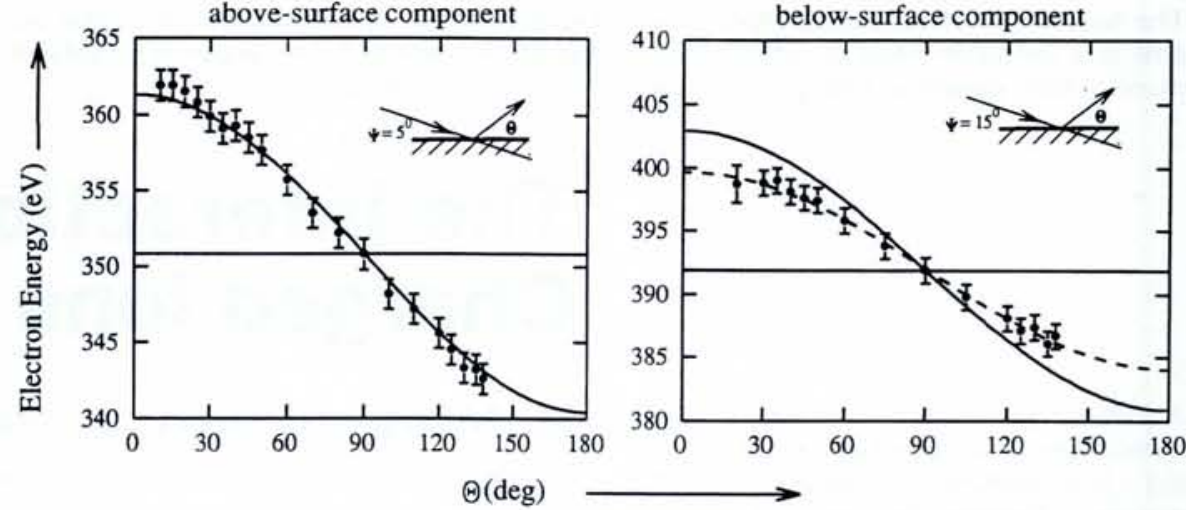

Fig. 4 - The measured angular dependence of the Doppler shifts for electrons in the abovesurface (a, left) and below-surface (b, right) structures of Fig. 3, compared with a calculated shift estimated assuming an undisturbed velocity of the electron emitting projectile.

which take place close to the surface. One of the most powerful involves measuring energy spectra of electrons ejected during Auger cascades. Since typical Auger decay times are on the order of tens of femtoseconds, dynamic processes occurring on a femtosecond time-scale are reflected in the electron energy spectra. The same is true for photon spectra provided that the characteristic times for photon emission are also on the order of tens of femtoseconds. Since hydrogenic lifetimes are on the order of nanoseconds and decrease roughly with $q^{-4}$ as the effective charge $q$ increases, photon decay competes with Auger decay on the same time-scale for charges $q=18$.

We [3] as well as other groups [4] have measured electron energy spectra in order to investigate the processes close to the surface. Fig 3 shows a spectrum obtained from collisions of hydrogen-like $\mathrm{N}^{6+}$ ions on a $\mathrm{Ni}(110)$ surface. The electrons near 400 $\mathrm{eV}$ can be ascribed to KLL Auger transitions, i.e., to the last step in an Auger cascade leading to the filling of the initial K-vacancy. But what are the fractions of the electrons ejected before and after touch down? Information can be obtained by measuring Doppler shifts because the Auger electrons are emitted from the projectile so their energy in the laboratory frame is influenced by the motion of the emitter. Measurements of energy shifts as a function of the observation angle therefore allow the motion of the projectile, both the absolute velocity and its direction, to be determined.

Fig. 4 compares calculated and experimentally determined Doppler shifts. In the calculations, it is assumed that electrons are emitted from the projectile moving with its initial energy and direction along a trajectory undisturbed by the interaction with the surface. The experimental values turn out to be different for different parts of the spectrum. For the sharp structure in Fig. 3 labelled as the "above-surface component" there is essentially perfect agreement between measured and calculated Doppler shifts (Fig. 4a). However, the measured shifts are significantly smaller for the majority of the electrons (Fig. 4b). This implies that a major part of the electron emission takes place after modification of the projectile velocity due to the interaction with the solid. For this reason the corresponding part in the spectrum is labelled "sub-surface component" (additional evidence justifying this labelling will be given later).

One can see from Fig. 3 that only a small fraction of the electrons is emitted above the surface. One could of course try to increase this fraction by decelerating the ions and thus increasing the time available for decay in front of the surface. But owing to the acceleration of the ions by their own image charge prior to neutralization, there is an intrinsic lower bound to the kinetic energy and thus an upper bound for the time available. $\mathrm{N}^{7+}$ ions have gained a kinetic energy of about $18 \mathrm{eV}$ before their neutralization starts at $D_{\mathrm{C}}=10 \AA$, and this leaves a maximum time of only about 70 fs until the touchdown. The influence of the image charge on the available time can be demonstrated directly by plotting the ratio of the abovesurface component to the total KLL Auger electron intensity as a function of the inverse of the initial projectile velocity (see Fig. 5).

One can clearly see that the above-surface fraction reaches a saturation value of about $10 \%$ at velocities around $10^{4} \mathrm{~m} / \mathrm{s}$. Lower initial velocities do not lead to a longer transit time through the critical region in front of the surface so they cannot yield an increase of the above-surface component. This implies that generally at most $10 \%$ of the initial inner shell vacancies have been filled when the ion hits the surface. Somewhat surprisingly, the projectile is still in a multiply excited state during touchdown.

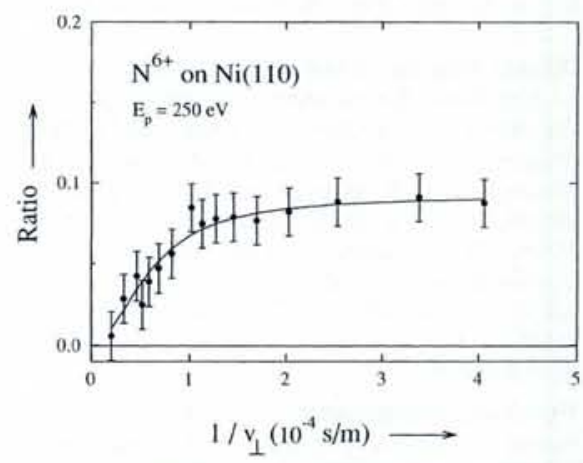

Fig. 5 - The fraction of the above-surface peak to the total KLL Auger electron intensity as a function of the inverse of the initial projectile velocity for $\mathrm{N}^{6+}$ colliding with $\mathrm{Ni}(110)$ at a projectile energy $E_{p}$ of $250 \mathrm{eV}$. 


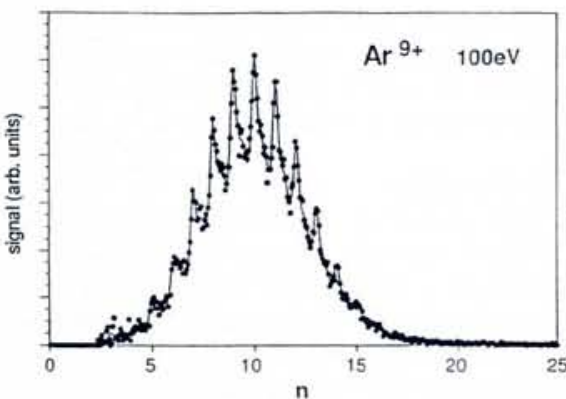

Fig. 6 - The statistical distribution of the number of electrons ejected during collisions of $100 \mathrm{eV} \mathrm{Ar}{ }^{9+}$ ions on a Au surface.

The structure of the electron spectrum of Fig. 3 and the shape of the saturation curve in Fig. 5 allows one extract further details about the processes taking place in front of the surface. Atomic structure calculations lead, for example, to the conclusion that the narrow above-surface peak is due to a decay of states with configurations in which only two electrons are in the L-shell of the neutralized projectile, while the other electrons are in. the $\mathrm{M}$ - or higher shells. The shape of the saturation curve in Fig. 5 allows quantitative determination of the time constant $\tau_{\text {av }}$ for the population transfer from the initially formed hollow atom to the configuration with two L-electrons, and of the constant $\tau_{\mathrm{K}}$ for the KLL Auger transition itself. The solid line in Fig. 5 represents in fact a fit which yields $\tau_{\mathrm{av}}=200$ fs and $\tau_{K}=70$ fs for these two characteristic time constants.

\section{Touch-down Phase}

The projectiles arrive at the surface in a neutralized state, but with many electrons in excited levels that have average distances from the nucleus significantly larger than typical screening lengths in a metal. When the projectile "dives" into the solid the excited electrons therefore loose contact with their core and can be regarded as being independently scattered from the surface. The electrons will often be reflected since their average kinetic energy is only fractions of an $\mathrm{eV}$. The projectile core, however, will penetrate into the solid so the excited electrons are "peeled off" and show up as slow, continuum electrons.

Winter's group, by developing an electron detector which is especially sensitive for low-energy electrons, has observed the emission statistics of such lowenergy electrons as a function of the projectile's initial charge and velocity [5]. Signals corresponding to single ion-surface collision events can be distinguished and are found to be proportional to the number of electrons released in each specific event. Fig. 6 shows the statistical distribution of the number of electrons emitted per collision, as measured for $\mathrm{Ar}^{9+}$ ions colliding on a gold surface. The group found rapidly increasing electron yields for increasing charge states, and it is clear that the Auger electrons showing up in our electron ener- gy spectra represent only a minor fraction of the total electron yield. Winter et al. found that the average electron yield is nearly independent of the projectile velocity over a wide range. This can be taken as indicating the release of the electrons during touchdown, since emission during Auger cascades in front of the surface should exhibit a strong velocity dependence of the yield owing to the variation of the time available for such cascades.

\section{Collisions within the Solid}

According to the scenario described in the previous sections, it is clear that the projectile ions carry a major fraction of their potential energy into the solid. Removal of the outer electrons during touch-down can be regarded as a resetting of an internal clock in the sense that the projectiles loose their memory of what happened during the precollision phase. After having entered the solid the projectiles undergo a whole series of close encounters with the target atoms; electrons can be directly transferred into the projectile's inner levels during these collisions. There is practically no restriction on possible impact parameters and therefore a large variety of states can be expected to be populated during these multiple collisions. This is reflected in the much larger width of the sub-surface component in Fig. 3 as compared to the above-surface component.

\section{Location of electron emission}

We have performed experiments with bare ions in order to obtain additional evidence about the location from which the electrons originate [6]. The initially empty projectile K-shells are in this case filled by two sequential KLL Auger processes leading to the emission of two electrons with different kinetic energies. Regarding subsurface emission, electrons from the second process will, on average, be ejected from a position deeper inside the solid than for the first. This should result in a stronger attenuation of their intensity as compared to electrons from the first process. The attenuation should become even more pronounced at detection angles for which the electrons have to traverse a longer path through the solid in order to reach the detec-

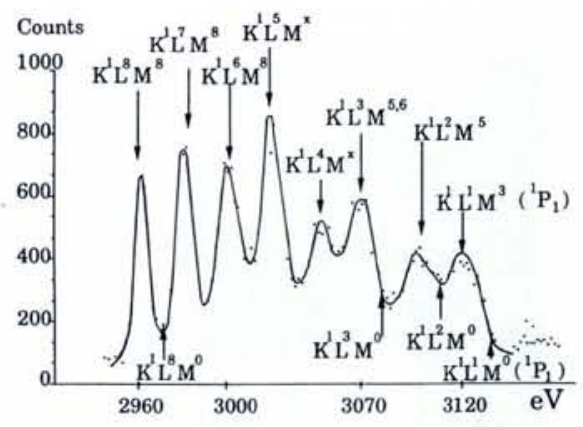

Fig. 8 - The spectrum of $X$-rays arising from collisions of $\mathrm{Ar}^{17+}$ on a $\mathrm{Ag}$ target: satellite lines corresponding to different $L$-shell filling are clearly distinguished.

tor. Fig. 7 shows parts of electron spectra arising from $\mathrm{C}^{6+}$ on a $\mathrm{Ni}$ surface for the three different collision geometries indicated in the upper part of the figure. The electrons are always observed perpendicular to the incoming projectile beam but the target crystal is rotated such that the projectiles hit the surface at different angles. For a near glancing angle of incidence (left-hand spectrum) the ions stay close to the surface for a long time and electrons observed at $90^{\circ}$ have to traverse only a short path through the solid. However, for increasing angles of incidence (right-hand spectra) the ions penetrate deeper into the solid and the electrons have to traverse a longer path through solid matter.

In the right-hand panels of Fig. 7 it can clearly be seen that the electron peak at lower energies, corresponding to the second KLL Auger process, suffers a significantly stronger attenuation on choosing a geometry which leads to a longer electron path through the solid. This represents strong evidence for sub-surface emission of most of the electrons.

\section{Shell filling}

It would of course be interesting to know more about the projectile states from which the electrons are emitted below the surface. For example, we would like understand how the various shells are filled. Unfortunately, the sub-surface electron spectra are rather broad and do not allow a detailed identification of these states. This is mainly due to inelastic scattering processes within the solid. However, in those cases where the inner vacancies are filled not only via Auger processes but also by radiative transitions, relatively direct information on the population of various states can be obtained from the measurement of X-rays, which leave the solid more or less undisturbed. K-shell fluorescence yields increase as $Z^{4}$ and become comparable to Auger yields around $Z=18$. One can therefore exploit X-ray measurements in experiments with hydrogen-like or bare $\mathrm{Ar}$ ions to gain more detailed information on the projectile states. Such measurements have been performed by Briand et al. [7], and Fig. 8 shows a typical spectrum obtained with $\mathrm{Ar}^{17+}$ 


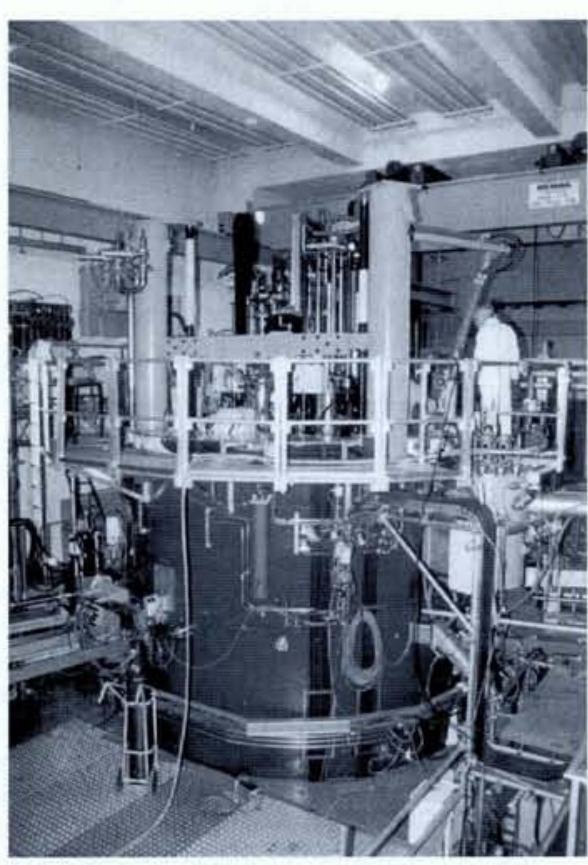

Research at the KVI (a joint venture between the University of Groningen and the FOM foundation) which makes use of light and heavy ions in various disciplines will receive a major boost with the installation of the second-generation, compact, superconducting, variable energy cyclotron AGOR. Shown fully assembled in the photograph, AGOR was built at the IPN Orsay and delivery to Groningen to replace the old (1972 construction) Philips cyclotron with a bending ratio $K$ of 160 is now scheduled for April or May following beam testing in Orsay. The $K=600$ machine will offer $200 \mathrm{MeV}$ protons, $400 \mathrm{MeV}$ alpha and $3 \mathrm{He}$ particles, $100 \mathrm{MeV} / \mathrm{u}$ light ions, and heavy ions down to $U$ ions of several $\mathrm{MeV} / \mathrm{u}$ for a multi-disciplinary facility. Nuclear physics will benefit from the higher energies (and polarized proton and deuteron beams) for studying nuclear excitations, nuclei in extreme conditions of temperature and angular momentum, and the influence of the nuclear medium on the nucleon-nucleon interaction. Atomic physics research on the interaction of multiply charged ions with different targets is to profit from the installation of a recoil-ion source that will deliver very slow ions with much higher charges than can be generated with ECR sources. A medical physics facility for proton therapy is also being considered. A Programme Advisory Committee has already been formed and M.H. Harakeh, Deputy-Director and responsible for the experimental scientific programme (the Director is R. Malfliet), emphasizes that external users as well as ideas for experiments in all fields are very welcome.

colliding with a $\mathrm{Ag}$ target. Lines corresponding to transitions from states with various L-shell fillings are clearly identified. The fact that the line at the lowest energy, corresponding to emission from a completely filled L-shell, is relatively strong implies that the filling of the L-shell is faster than its depopulation by radiative or Auger transitions. Auger cascades are unlikely to yield such a fast filling. In general therefore, changes of relative intensities of the various emission lines as a function of collision velocity and geometry can be used to test

\section{EPS on World-Wide Web}

The main information retrieval systems used on the all-pervasive Internet electronic communication network are Gopher, WIAS and WorldWide Web (W3). W3 is the newest and most powerful as it provides novel features based on combining hypertext and information retrieval techniques while offering access to Gopher and WAIS servers using a client-server paradigm. So W3 users can browse with ease through a global web of network-accessible information. Originally aimed at the high-energy physics community (with CERN as the instigator and driving force), W3 has spread into many other fields. While the number of W3 servers is today smaller than the 1000 or so available on Gopher, it is growing rapidly.

W3 consists of nodes (or documents) and links which are the connections between documents. To follow a link a user simply "clicks on" a highlighted word in a source document (or types in a number if a mouse cannot be used). This causes the linked-to document to be displayed. The document may be one of a variety of different media types, or it can be a searchable index of the sort used in Gopher. A Réseaux Associés pour la Recherche Européenne (RARE) report has recommended con- different scenarios for the population and depopulation of the L-shell.

For the case of bare projectile ions, the same type of X-ray experiments can also exploit the sequential emission of two photons, and Briand et al. [8] were in fact the first to apply this technique (they used bare $\mathrm{Ar}^{18^{+}}$ions). The two satellite spectra of sequentially emitted $\mathrm{Ly}_{\alpha}$ photons provide a measure of the L-shell filling at the corresponding times. L-shell filling rates within the solid could thus be monitored on a femtosecond time-scale. For Ar ions, Briand

centrating on W3 because of its flexibility, the availability of links, and the fact that it is an integrated system combining WAIS and Gopher support.

So W3 was the obvious choice for the Society's East-West Coordination Committee (EWCC) in making information available to physical societies and physicists throughout Europe. A W3 server has been set up by Eddy Lingeman with help from colleagues at CERN and at NIKHEF in Amsterdam; it is now running in the experimental mode with information on EPS, short items taken from Europhysics News, and some major documents such as the Directory of Physics Institutes in Central Europe published last year by EPS (available in soft-cover form from the EPS Secretariat; price: SFR 120.-)

To access the EPS information you need to call up your local W3 server with a command such as "www". A screen is displayed on which you select "high energy physics labs" with your mouse or by typing a number. Up comes another screen with a list of the labs. You select "NIKHEF" and on the NIKHEF screen you will find "EPS". Click it and you will see the EPS

- Client-browser terminal software for fetching and displaying data is available for many sysscreen. Some details as follows: et al. found that four additional electrons on average had entered the L-shell within the 20 fs which elapse between the two sequential Ly $\mathrm{L}_{\alpha}$ transitions.

\section{Conclusions}

The combined information on the collision of highly charged ions with surfaces, obtained from analyses of scattered ions as well as of ejected electrons and photons, has led to a detailed understanding of the electronic processes which take place near the surface. It is now clear that although the projectiles are neutralized at relatively large distances in front of the surface, they carry most of their potential energy into the surface layers of the solid. Surface modifications by highly charged ions via Coulomb explosion processes, for example, are therefore likely to occur. Indeed, recent experiments by our group involving the collision of multiply charged ions with $\mathrm{Si}$ surfaces demonstrate that for sufficiently low kinetic energies $(\approx 2 \mathrm{keV})$, the yield of ejected $\mathrm{Si}$ ions increases dramatically with increasing projectile ions (and with decreasing kinetic projectile energy). Intensive studies of the phenomena involved are foreseen for the future in view of possible technological applications for fabricating surface structures on a nanometre scale.

[1] Winter H., Europhys. Lett. 18 (1992) 207. [2] Burgdörfer J., Lerner P. \& Meyer F.W., Phys. Rev. A 44 (1991) 5674

[3] De Zwart S.T., Drentje A.G., Boers A.L. \& Morgenstern R., Surf. Sci. 217 (1989) 298; Das J. \& Morgenstern R., Phys. Rev. A 47 (1993) 755. [4] Meyer F.W., et al., Phys. Rev. A 44 (1991) 7214; Köhrbrück R., et al., Phys. Rev. A 45 (1992) 4653; Andrä H.J., et al., Suppl. Z. Phys. D 21 (1991) $\mathrm{S} 135$.

[5] Kurz H., et al., Phys. Rev. Lett. 69 (1992) 1140.

[6] Das J., Folkerts L. \& Morgenstern R., Phys. Rev. A 45 (1992) 4669

[7] Briand J.P., et al., Phys. Rev. Lett. 65 (1990) 159.

[8] Briand J.P., et al., Phys. Rev. A 43 (1991) 565.

tems including Macintosh, X/Motif, PC/MS Windows and NEXT; sever software is available for VM mainframes, UNIX, Macintosh, and VMS.

- All W3 compatible programs must be capable of handling the HyperText Markup Language as HTML provides one, simple format for providing linked information. W3 uses Internet's Hypertext Transfer Protocol (HTTP) to allow a browser program to request a keyword search by a remote user (i.e., to allow transfer representations to be negotiated between client and server).

- The W3 addressing mechanism means that an interface to Gopher, WIAS and anonymous FTP information sources may be established in a transparent way.

\section{For further information:}

- Telenet to "info.cern.ch" with user name "www" (no password): then follow instructions for the line-mode browser.

- The 1st International Conference on the Worid-Wide Web will be held at CERN on 2527 May 1994. Contact: Anne Perrelle, CERN, Geneva (tel.: +41-22-793-2406; fax: +41-22793-8730; email: www94@www1.cern.ch) or telnet to "www94.cern.ch" with logon "www94" (no password). 